\title{
Manifiesto para el desarrollo socialista en el siglo XXI
}

\author{
Benjamin Selwyn* \\ Mateo Crossa Niell**
}

Resumen. ¿Cómo podría ser el desarrollo socialista? Las principales concepciones del desarrollo consideran que la acumulación de capital es la base sobre la cual lograr el desarrollo humano. En estas concepciones de cambio, las clases trabajadoras se consideran un combustible para el motor del desarrollo, lo que a su vez justifica su explotación y opresión. En contraste, ¿cómo operar una estrategia de desarrollo socialista sin explotación? Este artículo ofrece un plan de 10 puntos para la transformación socialista sostenible.

Palabras clave: desarrollo, socialismo, Estado, sociedad, riqueza.

* Profesor investigador de Desarrollo Internacional en la Universidad de Sussex, Gran Bretaña.

" Doctorante en la Unidad Académica en Estudios del Desarrollo de la Universidad Autónoma de Zacatecas, México.

Este trabajo fue publicado en inglés como «A manifesto for socialist development in the 21st century», Economic \& Policy Weekly, 53(36), en septiembre de 2018. 


\section{Manifesto for the socialist development in the the 21st century}

Abstract. How could it be the socialist development? The principal conceptions of the development consider that the accumulation of capital is the ground on which the human development can be reached. In this conceptions of change, the working clases are considered fuel for the development engine, which at the same time justifies their exploitation and oppression. In contrast, how to operate a strategy of socialist development without exploitment? This article gives a plan of ten points for the sustainable socialist transformation.

Keywords: development, socialism, State, society, wealth. 
A principios de 2017 se reveló que ocho hombres poseían tanta riqueza como la mitad de la población mundial (Oxfam, 2017). Esto ocurre en un mundo donde, según las cifras más conservadoras, aproximadamente uno de cada tres trabajadores vive en la pobreza. Cálculos más realistas muestran que la mayoría de la población mundial sufre pobreza de una forma $u$ otra. ${ }^{1}$ Estas desigualdades y privaciones son sólo un síntoma del desarrollo capitalista. Otros incluyen la destrucción ambiental, el racismo sistemático y la discriminación de género, cada uno de los cuales genera sus propias cargas de pobreza.

Ya sea en el Chile de Augusto Pinochet (el laboratorio para desarrollo del mercado libre) o en la Corea del Sur de Park Chung-Hee (el caso más celebrado de desarrollo dirigido por el Estado), el desarrollo capitalista se basa en la explotación y la política de opresión del trabajo. ${ }^{2}$ Además, el desarrollo capitalista es predicado sobre la ruina ambiental y la (re)producción de diversas formas de discriminación.

Las teorías del desarrollo capitalista están unidas por la común concepción del trabajo como un recurso, o como un insumo en el proceso de desarrollo. Esta concepción es igual para el caso de los autodeclarados seguidores de mercado libre de Adam Smith, como para los seguidores estadistas de

${ }^{1}$ Según la Organización Internacional del Trabajo (OIT), en 2010 había aproximadamente 942 millones de trabajadores pobres (casi uno de cada tres trabajadores en el mundo vive con menos de 2 dólares por día) (OIT, 2013). La OIT calcula los niveles de pobreza utilizando las líneas de pobreza nominal extremadamente conservadoras del Banco Mundial de 1 y 2 dólares por día (paridad de poder de compra). Muchos expertos en pobreza argumentan que la línea de pobreza del Banco Mundial es demasiado baja, y recomiendan que se eleve de manera significativa, de modo que sea entre cuatro y 10 veces mayor (Edward, 2006; Sumner, 2016). En estos niveles, la mayoría de la población mundial vive en la pobreza.

2 Para la experiencia de desarrollo de Chile durante el gobierno de Pinochet, véase Marcus Taylor (2006) y para la experiencia de desarrollo de Corea del Sur bajo la administración de Park, véase Dae-Oup Chang (2002). 
Friedrich List (Selwyn, 2014; 2017). Tales teorías miran el mundo a través de la lente del capital y desempeñan un importante papel ideológico en el fortalecimiento del desarrollo capitalista al alentar que los pobres del mundo sigan esta misma mirada.

Tales perspectivas centradas en la mirada del capital se reproducen en al menos cuatro formas: $a$ ) identifican la acumulación de capital como base para el desarrollo de los pobres; $b$ ) identifican a las élites, corporaciones o Estados, como conductores de la acumulación de capital; c) grandes acciones, movimientos y luchas populares son mal vistos y a menudo se consideran obstáculos para el desarrollo; d) como consecuencia del punto anterior, la represión y la explotación son legitimados especialmente cuando esta última desafía al desarrollo centrado en el capital.

¿Es posible pensar en el desarrollo humano como un proceso que, más que profundizar la explotación capitalista, se base en trascenderla? ¿A qué se parecería la alternativa de una agenda de desarrollo socialista? ¿Podría resolver problemas de destrucción y deterioro ambiental y superar las diversas expresiones de discriminación? Sobre la base de un experimento de reflexión, el objetivo de este artículo es contribuir a tal conversación.

\section{Revolución intermitente ${ }^{3}$}

La conquista inicial del poder político por las clases trabajadoras no significará la trascendencia del capitalismo. Más bien representará una fase nueva y acentuada de la lucha por una transición a un modo de producción alternativo. Se

${ }^{3}$ El concepto de revolución intermitente se tomó de Cihan Tugal (2016). 
llevará a cabo utilizando herramientas heredadas del pasado: «Debe tenerse en cuenta que las nuevas fuerzas relaciones de producción no se desarrollan de la nada, ni caen del cielo, ni del útero de una idea auto posicionada; sino desde dentro de una antítesis al desarrollo existente de la producción y de las relaciones heredadas y tradicionales de la propiedad» (Marx, 1993:278).

Habrá numerosas empresas donde aún existan relaciones capital-trabajo. Un gran número de trabajadores desempleados buscarán trabajo e ingresos. Aun así, con toda probabilidad, los hogares serán liderados por mujeres, dependientes de los ingresos basados en el trabajo y orientados hacia (re)producir las generaciones actuales y futuras de trabajadores. La mayoría de la tierra probablemente será poseída por una pequeña minoría de agricultores capitalistas y terratenientes. El comercio exterior se producirá en términos capitalistas. Las instituciones financieras y su poder dentro de la economía permanecerán altamente concentradas. Las discriminaciones de género, raciales y étnicas seguirán existiendo. Las instituciones democráticas serán disfuncionales desde la perspectiva de establecer una sociedad genuinamente participativa.

Bajo tales circunstancias, las políticas y estrategias de un Estado socialista emergente necesitan expandir y mejorar simultáneamente el dinamismo del poder de la clase trabajadora, mientras se reduce el poder del capital. Se requerirá mucho tiempo para subordinar las relaciones sociales capitalistas a las relaciones socialistas. Precisamente, debido a este extenso proceso cargado de contradicciones, es doblemente necesario considerar cómo un Estado emergente de clase trabajadora puede mantener el entusiasmo y la energía iniciales de las clases que lo han creado, facilitar su reproducción social mejorada y contribuir, en un tiempo futuro desconocido, para la expansión global del desarrollo humano socialista. 
El proceso de mejorar el poder de la clase trabajadora puede ser considerado como una revolución intermitente (Tugal, 2016). Estas transformaciones se producirán a corto, mediano y largo plazos, y adoptarán muchas formas, incluida la construcción de instituciones alternativas (cooperativas y comunas); medios alternativos para asegurar y ampliar los medios de supervivencia (la producción y distribución de alimentos y otras necesidades básicas); nuevos sistemas de educación participativa y la acumulación a medio y largo plazos de la experiencia política (de defender y extender el poder de la clase trabajadora). Una política internacionalista que mira hacia el exterior puede complementar la extensión interna del poder de la clase trabajadora a través de la colaboración con los movimientos sociales internacionales para construir la solidaridad y el apoyo hacia el nuevo régimen, especialmente defenderlo de la intervención hostil y, cuando surjan oportunidades, extender el proceso hacia la internacionalización del fortalecimiento del poder de la clase trabajadora.

El surgimiento inicial y el establecimiento de un Estado de clase trabajadora democrática en un país es la condición previa para el surgimiento de otros Estados similares; el advenimiento de este último es necesario para preservar la fortaleza del primero a largo plazo. Con toda probabilidad, habrá un importante lapso entre la aparición del primer Estado de este tipo y su multiplicación global. Es dentro de este tiempo que una estrategia de desarrollo socialista debe ser formulada e incentivada. 


\section{Reabsorción del Estado por la sociedad ${ }^{4}$}

Después de estudiar la Comuna de París, Karl Marx argumentó que esta era «la forma política que por fin se descubrió bajo la cual resolver la emancipación económica del trabajo», ya que «serviría como una palanca para desenraizar los cimientos económicos sobre los que se basa la existencia de clases, y por lo tanto del dominio de clase» (1966). Caracterizó el proceso radical de cambio de las relaciones sociales y, en particular, la relación del Estado con la sociedad como «[1]a reabsorción del poder del Estado por la sociedad como sus propias fuerzas vivas en lugar de como fuerzas que la controlan y la subyugan, por las masas populares mismas, formando su propia fuerza en lugar de la fuerza organizada de su opresión - [es] la forma política de su emancipación social» (Marx, 1966).

Se requiere la reabsorción social del Estado para subordinar y transformar las relaciones sociales capitalistas. Tres principios organizativos pueden contribuir a pensar cómo podría ocurrir un proceso de transformación de este tipo (Lebowitz, 2015).

Propiedad social de los medios de producción. «La producción [capitalista] de mercancías ha sido la forma social en la que se ha logrado el sistema de interdependencia social más desarrollado de la historia humana» (Barker, 1998:3). Sin embargo, los medios de producción están dirigidos de manera autocrática, de acuerdo con los imperativos del mercado en la competencia de la acumulación de capital. Dichas estructuras de propiedad privan a los trabajadores de cualquier opinión sobre cómo y hacia qué fin se orienta la producción y los reduce a «objetos» para ser manipulados

${ }^{4}$ En gran medida, esta sección se basa en los aportes de Michael A. Lebowitz (2015:183-184) y Marta Harnecker (2014). 
por «sujetos» gerenciales. La propiedad social de los medios de producción, por el contrario, reconstituiría la toma de decisiones como un proceso democrático colectivo.

Producción social dirigida por el trabajo. La propiedad social de los medios de producción facilita la dirección social de la producción a través de la cooperación entre los trabajadores y la comunidad. Dicha cooperación es una propiedad esencial de una sociedad socialista emergente por dos razones. Primero, porque limita, reduce y eventualmente elimina la producción basada en la competencia autocrática y anárquica. Segundo, porque la fuerza vital del desarrollo socialista es la cooperación (dentro y fuera de los lugares de trabajo).

Identificación y satisfacción de las necesidades y propósitos de la comunidad. En el capitalismo, las empresas rivales compiten para obtener mayores ventajas competitivas. Los hogares de la clase trabajadora y los individuos compiten entre sí para asegurar los mejores trabajos. Las organizaciones de base comunal, organizadas dentro y fuera de los lugares de trabajo, representan una lógica alternativa de reproducción social. La identificación y satisfacción de las necesidades y propósitos de la comunidad se basarán en la cooperación dentro y entre los lugares de trabajo y las comunidades.

¿Cómo se pueden poner en práctica estos principios organizativos? Un proceso de planificación participativa descentralizada en el nivel local representa un método posible (Harnecker, 2014). Bajo tal sistema, la energía social generada por la planificación (elaboración y ejecución de un plan) fluye hacia arriba, desde el nivel local hasta el nacional, en lugar de sólo hacia abajo por empresas y Estados, como en el caso del capitalismo. Un principio que informa este proceso es que «todo lo que se puede hacer en el nivel 
inferior debe descentralizarse a este nivel» (Harnecker, 2014). La economía nacional será reorganizada hacia el logro de estos objetivos. Las necesidades y los objetivos que no se pueden cumplir en el nivel local se transmitirán hacia arriba, a los organismos de planificación superiores, que pueden incorporarse en la generación más amplia de recursos y estrategias de asignación.

El establecimiento y la transmisión hacia arriba de los impulsos de planificación democrática requieren escalas apropiadas de planificación participativa. Dichas escalas, diferentes pero interdependientes, pueden estar constituidas por comunidades vecinas, comunas, consejos municipales y organismos nacionales (Lebowitz, 2015).

Dentro de una comunidad, los vecinos pueden reunirse regularmente para discutir en qué tipo de comunidad quieren vivir, y luego identificar y coordinar las necesidades y capacidades de las comunidades para satisfacer esas necesidades. La probabilidad de una coincidencia precisa entre las necesidades de la comunidad y la capacidad de satisfacer esas necesidades es pequeña. El propósito de la planificación a escala local es, en parte, identificar y comunicar hacia arriba qué recursos adicionales se requieren y qué capacidades adicionales están disponibles.

La comuna representa la siguiente escala de planificación participativa descentralizada. Combina diversos barrios y lugares de trabajo. La información de las comunidades se reúne y se discute dentro de los lugares de trabajo. ¿Pueden los trabajadores satisfacer las necesidades de las comunidades que conforman la comuna? Bajo el capitalismo, donde la producción es la orientación hacia la generación de valores de cambio (para la generación de ganancia mediante la venta de valores en los mercados), tales consideraciones — si es que las hay - son secundarias a las de la maximización. En una sociedad socialista emergente, la identificación de intentos de satisfacer las necesidades 
locales comienza a producir la sustitución de los valores de cambio por los valores de uso (bienes producidos para satisfacer las necesidades de clase) . A través de reuniones comunales los consejos pueden generar datos sobre:

a) Necesidades que pueden ser y son satisfechas por y dentro de la comunidad y la comuna; b) necesidades que no pueden ser satisfechas por la comunidad — que necesita el apoyo de la comuna y más allá—; c) capacidad excedentaria de los lugares de trabajo — que puede contribuir a satisfacer las necesidades de otras comunidades y comunas.

La capacidad excedentaria y las necesidades no satisfechas se comunican más adelante en la cadena de planificación participativa hacia unidades de mayor escala, desde las ciudades comunales hasta el Estado nacional. A medida que las comunas elaboran su lista de necesidades, sus limitaciones para satisfacerlas y sus capacidades excedentes, la comunidad estatal de nivel nacional puede evaluar cómo generar y asignar recursos. Donde haya necesidades excesivas, las discusiones girarán en torno a mecanismos para aumentar la producción, la reasignación (regional o social) de recursos o las posibilidades de reducir la satisfacción de algunas necesidades.

A través de la planificación participativa descentralizada, los participantes obtienen conocimientos sobre la disponibilidad, producción y asignación de recursos. En su análisis de las experiencias de planificación participativa descentralizada en Brasil, Venezuela e India, Marta Harnecker escribe cómo representa un doble proceso:

Un primer producto objetivo material: el plan construido en forma participativa que es algo palpable porque está a la vista de todos, y un segundo (...) la transformación de las personas a través de esa práctica. Se trata de un proceso educativo en el que quienes participan van aprendiendo a indagar las causas 
de las cosas, a respetar las opiniones de los otros, a entender que los problemas que confrontan no son exclusivamente de su calle y de su barrio, sino que están relacionados con la situación global de la economía, la situación social nacional, inclusive con la situación internacional (...) con todo eso se van creando nuevas relaciones de solidaridad, de complementariedad, que ponen el acento más en lo colectivo que en lo individual (2014:8).

La planificación participativa descentralizada requerirá cierta coordinación central y, en última instancia, el poder para determinar la asignación de recursos. Su extensión no se puede determinar de manera abstracta y dependería de consideraciones que van desde variaciones en las capacidades de diferentes comunidades para satisfacer sus necesidades, hasta circunstancias globales cambiantes.

\section{Recuperando la riqueza social}

El argumento central en esta sección es que la redistribución de la riqueza a través de la transformación de las relaciones sociales representa el medio más rápido para aliviar la pobreza y, al hacerlo, establece posibilidades y procesos de desarrollo humano genuinamente progresivos. A menudo se afirma que si bien esa redistribución contribuiría a un desarrollo humano significativo en los países ya ricos (donde el pastel a redistribuir es relativamente grande), es poco probable que lo haga en países relativamente pobres. Más bien, estos países necesitan acumular riqueza antes de redistribuirla y, en consecuencia, deben pasar por un proceso de rápido desarrollo capitalista. El desarrollo no capitalista es, por lo tanto, excluido por una o muchas generaciones. 
Tales argumentos con frecuencia dan por sentado, o simplemente ignoran, las formas en que las clases capitalistas en los países pobres pueden acumular riqueza, a menudo en alta mar, y protegerla de los impuestos nacionales y del uso potencialmente determinado democráticamente. Por ejemplo, un estudio reciente de Léonce Ndikumana y James K. Boyce (2011) muestra cómo:

África subsahariana experimentó un éxodo de más de \$700 mil millones en fuga de capital desde 1970 (...) África es un acreedor neto para el resto del mundo en el sentido de que sus activos extranjeros superan sus pasivos externos. Pero hay una diferencia clave entre los dos: los activos están en manos de los africanos privados, mientras que los pasivos son públicos, controlados en general a través de sus gobiernos.

Esto se compara con los $\$ 177$ mil millones en deudas externas de África (Ndikumana y Boyce, 2011). James S. Henry (2012), Nicholas Shaxson et al. (2012), James K. Boyce (2011) proporcionan datos para 139 «países con ingresos en su mayoría de ingresos medios bajos» y señalan que:

Los datos tradicionales muestran deudas externas agregadas de $\$ 4.1$ billones a fines de 2010. Pero teniendo en cuenta sus reservas extranjeras y la riqueza privada extranjera no registrada, el panorama se revierte: tenían deudas netas agregadas de menos de \$10.1-13.1 billones (...) Estos países son grandes acreedores netos, no deudores. [Sin embargo], sus activos son mantenidos por unos pocos individuos ricos, mientras que sus deudas son asumidas por la gente común a través de sus gobiernos. ${ }^{5}$

${ }^{5}$ Para consultar el reporte véase James S. Henry (2012), Nicholas Shaxson et al. (2012) y James K. Boyce (2011). 
Deborah Rogers y Bálint Balázs (2016) demuestran que en países muy pobres una distribución relativamente pequeña de la riqueza de ricos a pobres podría eliminar la pobreza: «Usando números que se aproximan a los de Bangladesh en 1995-1996, una redistribución de 3 por ciento de los ingresos del quintil superior (reducido de 40.2 a 37.2 por ciento) al quintil inferior (elevado de 9.3 a 12.3 por ciento) resulta en una reducción de 20 a 0 por ciento en pobreza extrema». Continúan: «Intentar reducir la pobreza en una cantidad similar mediante el crecimiento de la economía requiere una expansión del ingreso total de aproximadamente 45 por ciento» (Rogers y Balázs, 2016:62).

De manera similar, Chris Hoy y Andy Sumner muestran que aun el ejercicio de una redistribución limitada de la riqueza — por ejemplo, mediante la redirección de los subsidios a los combustibles de sus beneficiarios relativamente acomodados a los pobres - puede tener efectos significativos: «la mayoría de los países en desarrollo tiene la capacidad financiera para poner fin a la pobreza (...) elevando un ingreso de 1.90 o 2.50 dólares hacia potencialmente 5 dólares por día (Hoy y Sumner, 2016:3). En estos cálculos se utilizan definiciones más bien conservadoras (basadas en el dinero) de la pobreza. Además, estos cálculos suponen una redistribución de la riqueza limitada dentro de las estructuras sociales capitalistas que aún existen.

Nuestra concepción del desarrollo socialista implica una concepción más amplia, social, de la riqueza. Incluye no sólo el ingreso y el dinero, sino también los medios para generar riqueza social, desde la tierra y los lugares de trabajo hasta el entorno natural. Bajo el capitalismo, esta riqueza se produce socialmente pero es de propiedad privada. Nuestros objetivos son transformar, de manera radical, la producción de la riqueza de la sociedad a través de la socialización de su propiedad y su dirección democrática. 
La distribución de la riqueza monetaria representa un primer paso necesario para eliminar la pobreza. Sin embargo, tales medidas tienen sus límites, ya que la distribución de la riqueza requiere su producción previa. ¿Cómo podría una organización y distribución socialista de la producción de riqueza social contribuir a mejorar aún más las condiciones de la población de un país pobre?

\section{Plan de 10 puntos}

A continuación se presenta un plan de 10 puntos para la construcción de un desarrollo socialista. Antes de continuar se debe afirmar, con 100 por ciento de claridad, que cada caso de desarrollo socialista será diferente, dependiendo de la base de recursos, incluidos los niveles de pobreza, la constitución particular del poder de la clase trabajadora, las alianzas políticas, y fundamentalmente si son desarrollos socialistas de tardía o reciente creación —es probable que estos últimos se encuentren en una situación internacional más favorable debido a la asistencia de desarrolladores socialistas anteriores.

Si bien cada forma de desarrollo socialista será histórica, geográfica y socialmente específica, dada la extensión global del capitalismo, se enfrentará a retos similares. El poder del capital tendrá que ser desmantelado, de una manera que no destruya a la sociedad. El desafío será utilizar lo que está disponible, heredado del pasado capitalista, para construir algo nuevo, un futuro socialista.

Muchas de las propuestas que se sugieren a continuación son, en ausencia de una transformación social más amplia, compatibles con el capitalismo. 
Algunos de ellos ya han sido implementados. Si estas políticas son compatibles con el desarrollo capitalista contemporáneo, entonces, ¿por qué y cómo podrían contribuir al desarrollo socialista? Que una política contribuya al desarrollo capitalista o socialista depende de las relaciones sociales dentro de las cuales se produce y de los objetivos a los que sirve. Las políticas pueden ayudar a engendrar el desarrollo socialista si contribuyen a la transformación radical de las relaciones sociales. Las políticas progresistas en ausencia de transformación social dejarán el poder capitalista intacto, listo y capaz de socavar la organización de la clase trabajadora.

\section{Bancos, dinero y democracia económica}

El dinero y los bancos privados no representan medios naturales e instituciones de intermediación financiera. Por el contrario, contribuyen directamente a la dinámica de crecimiento del capitalismo, a la diferenciación de clase y regional, así como a la concentración del poder capitalista. El dinero y los bancos son recursos sociales que pueden ser públicos o privados. Pueden atender necesidades tanto democráticas como autocráticas. El sistema financiero global actual no es simplemente un mecanismo a través del cual se asigna el dinero. Más bien, es un sistema de poder que garantiza flujos continuos de recursos globales hacia el régimen de dollar-Wall Street (Gowan, 1999).

El primer objetivo será cancelar lo que consideramos deudas odiosas (deudas contraídas por la administración anterior en beneficio de las clases capitalistas en lugar de las trabajadoras). Introduciremos controles de capital. Dichos controles, determinados e implementados por un Estado de clase trabajadora regularán el movimiento de capital dentro y fuera del país, y son necesarios para generar estrategias de desarrollo socialista (Crotty y Epstein, 
1996). Dichos controles regularán la exportación de dinero y finanzas (para evitar fuga de capital y someter el capital nacional a los imperativos democráticos nacionales). También servirán para orientar la inversión extranjera hacia empresas socialmente dinámicas y beneficiosas, potencialmente en colaboración con empresas locales. A medida que se cierran las opciones de fuga de capital (que utiliza para obtener concesiones de mano de obra), los recursos generados en el país que aún se encuentran en manos privadas se invertirán en el país, en condiciones determinadas democráticamente.

En el capitalismo, los bancos efectivamente crean dinero a través de préstamos (el llamado «dinero a primera vista») (Mellor, 2005). Estas cuentas requieren crecimiento para pagar intereses (que generalmente son más bajos para aquellos que ya han acumulado grandes reservas de dinero y más altos para aquellos que no tienen dinero). Los bancos centrales y los Estados aplican el poder de los bancos privados mediante la regulación de la oferta monetaria para garantizar que los trabajadores sólo puedan obtener dinero mediante la venta de su fuerza de trabajo, préstamos (basados en intereses) o con muy limitadas prestaciones sociales.

Bajo el capitalismo, la escasez es una consecuencia de las relaciones de clase, de la falta de control de los trabajadores sobre los medios para producir riqueza social. Una sociedad cada vez más democrática puede comenzar a eliminar esta escasez al socializar las finanzas, al integrarlas en estructuras cooperativas emergentes y al reemplazar gradualmente el dinero derivado de los salarios con una subvención/ingreso básico universal (Standing, 2017).

El dinero será cada vez más conceptualizado y funcionará como un recurso público y como instrumento de desarrollo socialista (Mellor, 2012). Un nuevo sistema de contabilidad, que abarca asociaciones en los niveles local y nacional, calculará a) las necesidades básicas y extendidas de la 
población, que van desde el consumo de alimentos hasta los requisitos de desarrollo de infraestructura; b) los recursos disponibles de la nación. El dinero se distribuirá a través de cuentas bancarias estatales a individuos y asociaciones, para que los recursos de las sociedades, desde la materia prima hasta la mano de obra, coincidan con los requisitos/necesidades determinados democráticamente.

En lugar de que el estado dependa de los impuestos para recaudar e invertir dinero, el dinero se invertirá sobre la base de cálculos de la necesidad determinada por la democracia y la disponibilidad de recursos. Si se distribuye demasiado dinero, lo que potencialmente lleva a la inflación, los impuestos públicos se utilizarán para reducir la oferta monetaria. Los bancos comerciales restantes se transformarán en intermediarios entre los depositantes y los prestatarios, y sus costos operativos se cubrirán con las tarifas de los usuarios.

\section{Ingreso básico universal}

La explotación capitalista se produce porque la clase trabajadora carece de los recursos (como el dinero y la tierra) para mantenerse y está obligada a vender su fuerza de trabajo por salarios. Un ingreso básico universal (IBU) puede contribuir a eliminar esta coacción, la construcción de una economía política solidaria y la socialización de la reproducción del trabajo. También, de inmediato, aliviará muchas formas de privación y de pobreza.

Las transferencias monetarias en los países pobres han ayudado a combatir la pobreza. Por ejemplo, en la primera década de este siglo, los programas de transferencia en Malawi ayudaron a aumentar la asistencia escolar entre las niñas en 40 por ciento, y en Namibia redujeron la desnutrición (de 42 a 10 
por ciento) y el absentismo escolar (de 40 a casi 0 por ciento). El IBU es asequible incluso para Estados con presupuesto inicialmente limitado y grandes poblaciones pobres. Reducir o eliminar los subsidios a las empresas que no producen para el bien social determinado democráticamente, así como a los sectores de la población en mejores condiciones, puede financiar tales subsidios inicialmente (Bardhan, 2016).

El IBU funcionará adecuadamente con una condición necesaria. Cada persona adulta sin discapacidad tendrá el deber de realizar un trabajo doméstico no remunerado dentro de sus comunidades para apoyar y cuidar a quienes no pueden cuidarse a sí mismos. Sólo aquellos que ya lo hagan estarán exentos de la condición. La riqueza y los recursos existentes generarán, a través de la redistribución, la provisión pública cada vez más gratuita de actividades de cuidado (como guarderías, hogares de ancianos, comedores comunales e instalaciones básicas de salud). El IBU complementará dichos arreglos de cuidado y contribuirá a la reestructuración de las relaciones de género al reconocer y distribuir socialmente este trabajo entre la población masculina y al reducir la cantidad de trabajo reproductivo doméstico de las mujeres (Elson, 1988).

\section{Políticas industriales para una transformación ecológica}

La propiedad social y la dirección de la industria contribuirán a establecer el desarrollo socialista. El socialismo radical de la Unión Nacional de Trabajadores Metalúrgicos de Sudáfrica (NUMSA) sostiene que la forma más efectiva de democratizar la economía sudafricana es con la nacionalización del lucrativo sector minero. Se basa en la Carta de la Libertad de 1955: 
¿La gente compartirá la riqueza del país! La riqueza nacional de nuestro país, la herencia de los sudafricanos, será restaurada a la gente; la riqueza mineral debajo del suelo, los bancos y la industria del monopolio serán transferidos a la propiedad de la gente en su conjunto. Todas las demás industrias y comercios deben ser controlados para ayudar al bienestar de las personas; todas las personas tendrán los mismos derechos para comerciar donde elijan, para fabricar e ingresar a todos los oficios que escojan, manufactureros y profesiones (SAHO, 2011).

Una política industrial socialista apunta a transformar la manufactura, fuera del valor de cambio con fines de lucro y hacia la producción de valores de uso, para satisfacer las necesidades de los trabajadores y de las comunidades en general. La transformación se manejará para mantener algunos ingresos en divisas que permitan comprar bienes esenciales que no se pueden producir localmente. También apuntará a dejar la manufactura de los combustibles fósiles y a encaminarla hacia la producción basada en energía renovable a través de inversiones en esta última. Las industrias orientadas a la exportación serán dirigidas por consejos de trabajadores, integrados en organizaciones de planificación descentralizadas.

Nuestra política industrial buscará generar una combinación adecuada de actividades de alta y baja tecnología enfocadas a la satisfacción de las necesidades básicas y ampliadas. Las inversiones a gran escala se orientarán hacia la generación de un sistema nacional de energía ecológica, que comprende una combinación de tecnología solar a pequeña escala y turbinas eólicas a gran escala conectadas a una red nacional.

La investigación y el desarrollo industrial (I+D) de bajo uso tecnológico se centrarán en áreas como la producción y la distribución generalizada 
de estufas (como estufas cohete), purificadores de agua de cerámica, dispositivos de desalinización que funcionan con energía solar, sistemas de inodoros, iluminación (como luces de gravedad), duchas calentadas con energía solar, bombillas de energía solar, sistemas de refrigeración de dos vasijas, bombas de agua que funcionan con bicicletas.

Los cambios tecnológicos de gama alta incluirán la transformación de autopartes en fábricas de bicicletas, autobuses y trenes; productos de belleza en productos farmacéuticos destinados a la salud; publicidad en la educación popular; y producción de armas en producción de aparatos domésticos.

La articulación intersectorial entre la industria y la agricultura aumentará la productividad en la agricultura y establecerá un subsector industrial dinámico, innovador y adaptable. Se establecerán foros de productores agrícolas e industriales para identificar los desafíos y las formas de enfrentarlos, por ejemplo, a través de inversiones en biotecnología que mejoren el rendimiento.

El Estado invertirá en la creación de talleres de pequeña escala en las comunidades locales. Dichas inversiones harán posible la expansión de las economías locales sustentadas en tecnologías apropiadas. Los talleres comunitarios permitirán la producción en el nivel local de muchas cosas que antes sólo eran accesibles a través de la compra. También servirán como centros de reciclaje aquellos lugares para el intercambio de excedentes e intercambio de información (Trainer, 1996). La producción en masa y la distribución de bicicletas, así como la construcción de carriles para bicicletas a lo largo de los espacios urbanos y rurales, quitarán estímulo al uso de automóviles que funcionan con combustibles fósiles.

Las inversiones estatales en I+D facilitarán la transferencia de tecnología y conocimiento. Éstos serán facilitados y alentados por formas de 
intercambio no comerciales, como el acceso abierto y las relaciones entre pares (los ejemplos contemporáneos incluyen Wikipedia, Copyleft y varias formas de software de código abierto).

\section{Reforma agraria}

La concentración global de la tierra es producto del imperialismo, el imperativo del mercado capitalista y el apoyo estatal al capital basado en la propiedad privada de tierra (Akram-Lodhi, 2015). Esta concentración y el «modelo» agroindustrial orientado hacia la exportación niegan el acceso de los trabajadores a la tierra y sostienen la existencia y expansión de una población excedente, desempleada. También es un factor causal en la «paradoja» de la escasez, falta de alimentos para grandes segmentos de los pobres del mundo, en un mar de abundancia (sobreproducción global) (McMichael, 1994).

Los objetivos de una reforma agraria son: a) contribuir al logro de la seguridad alimentaria nacional, donde se produzcan los alimentos suficientes para satisfacer las necesidades de la población; $b$ ) para generar empleo de alta calidad. En contraste con los ejemplos anteriores de reforma agraria pro-capitalista, estos objetivos sirven al objetivo de des-mercantilizar la tierra, los alimentos y los recursos naturales y, al hacerlo, establecer una sociedad donde el consumo adecuado de alimentos se convierta en un verdadero derecho humano.

Tales objetivos y metas existen dentro de un sistema de restricciones. En particular, la agricultura de exportación a menudo genera divisas para las importaciones necesarias que aún no pueden producirse en el país. Por tanto, al igual que la estrategia industrial, las propuestas de reforma agraria 
se basan en una concepción de construcción de un sistema agrario mixto. Las reformas inmediatas incluirán la transformación de la propiedad de grandes concentraciones privadas orientadas a la exportación y controladas por capitalistas, a cooperativas de trabajadores. Estas cooperativas, junto con los objetivos nacionales, combinan producción para la exportación e intercambio en el mercado extranjero, con producción encaminada al consumo nacional.

Se preservará el sector de la agricultura familiar a pequeña escala, pero la tierra dejará de ser un producto vendible. El ingreso básico universal brindaría seguridad social a los trabajadores y agricultores familiares en momentos en que no pueden producir. Las tierras comunales serían preservadas y expandidas.

El objetivo de lograr una seguridad alimentaria des-mercantilizada, en la cual los alimentos sean un derecho humano básico, independiente del poder de compra, se buscará por medio de inversiones multinivel, desde comunidades locales hasta el nivel nacional, con el fin de mejorar la productividad agrícola sostenible y de bajos insumos. También se impulsará con la combinación de I+D con alto y bajo contenido tecnológico. El desarrollo de I+D con bajo contenido tecnológico incluye la facilitación, la construcción y la conservación de la fertilidad del suelo; el uso de controles biológicos para enfermedades, insectos y malezas; cultivos intercalados; ahorro y selección de semillas; ciclos de recolección múltiple a pequeña escala; y la integración de pastoreo y pastoreo a pequeña escala (Weis, 2010:334). Por su parte, el desarrollo de I+D de alto contenido tecnológico implica aumentar la productividad a través del desarrollo de nuevas variedades de plantas. Como lo sugiere Jack Kloppenburg (2010:379), «el fitomejoramiento participativo ofrece una modalidad a través de la cual la fuerza de trabajo de millones de agricultores 
se puede combinar sinérgicamente con las habilidades de un grupo mucho más pequeño de fitomejoradores».

La reforma agraria se extendería a los centros urbanos. Los edificios no utilizados pueden transformarse en invernaderos; los techos pueden usarse como nuevos espacios de crecimiento; las carreteras innecesarias pueden convertirse en campos, parcelas y parques; la jardinería doméstica se fomentará y facilitará mediante la provisión de insumos, tecnologías y educación en permacultura. Ted Trainer (1996:139) explica: «La mayoría de este espacio urbano puede (...) convertirse en huertos para la permacultura, densamente poblados con plantas, principalmente perennes, de modo que los asentamientos tengan fuentes permanentes de alimentos y muchos insumos para proveer a la producción artesanal».

\section{Protegiendo y aprendiendo de los pueblos indígenas}

Desde «el descubrimiento» de América en 1492 hasta la globalización contemporánea, el acaparamiento de tierras, el despojo de los pueblos indígenas y el despojo de los entornos naturales han sustentado la expansión geográfica del capitalismo (Clarke y Foster, 2009). Sin embargo, los pueblos indígenas han estado con frecuencia en la vanguardia de la oposición a la expansión y depredación capitalista, con la búsqueda de formas alternativas de vivir en conjunto con el entorno natural. Joan Martínez-Alier (2003) se refiere a estas luchas como el ambientalismo de los pobres. Al preservar sus derechos a la tierra y la cultura, un Estado socialista emergente también establecerá foros para compartir el conocimiento y la práctica entre las comunidades. La protección y la preservación del derecho de los pueblos indígenas a vivir de acuerdo con sus prácticas será necesaria en la concepción del desarrollo socialista. 
En distintas partes de América Latina, el discurso y la práctica de Sumak Kawsay o Buen Vivir representan una concepción alternativa, potencialmente anticapitalista del desarrollo humano. Se aboga por «vivir en plenitud, saber cómo vivir en armonía con los ciclos de la Madre Tierra, del cosmos, de la vida y de la historia, y en equilibrio con todas las formas de existencia en un estado de respeto permanente» (Mamani, 2010:32).

\section{Política exterior}

La política exterior se basará en un doble enfoque. Por un lado, el principio rector de las relaciones externas es la no agresión y la búsqueda de la coexistencia pacífica con los poderes capitalistas. Por otro lado, estableceremos vínculos con movimientos sociales en todo el mundo que se esfuerzan por transformar sus sociedades. La asistencia a estos movimientos consistirá en el efecto demostración. La información y práctica sobre los éxitos a corto plazo se difundirá y ayudará a los movimientos sociales a interpretarlos en el contexto de objetivos transformadores sociales a largo plazo.

Se intentará participar en debates internacionales sobre estrategias de desarrollo alternativo, promover la experiencia y explicar su posibilidad y el alcance de su aplicación en otros lugares. Tres serán los objetivos: 1. Fortalecer los movimientos sociales transformadores globales para ayudarlos a lograr sus propósitos. 2. Generar presiones de clase trabajadora sobre los Estados capitalistas progresistas (es decir, los Estados «gobernados» por los partidos progresistas) con la intención de que nos brinden asistencia para el desarrollo. 3. Facilitar presiones similares desde abajo para impedir las intervenciones de Estados capitalistas hostiles diseñadas en aras de socavar la agenda transformadora. 
También se espera que, a mediano y largo plazos, otros Estados se someterán a un proceso complementario de transformación social y se integrarán en una comunidad social global. El conocimiento y los recursos se construirán y transferirán entre los Estados progresistas.

\section{Política económica exterior}

Como parte de la política económica exterior, se buscará que la comunidad internacional genere una agenda colectiva para combatir la destrucción del medio ambiente. La perspectiva adoptará el punto de vista, en primer lugar, del Movimiento por la Justicia Climática (MJC) que se estableció como un movimiento en contra del Protocolo de Kioto dominado por los países ricos y la agenda ambiental global del comercio de carbono, diseñado para legitimar la expansión industrial basada en combustibles fósiles.

Creemos que la siguiente propuesta del MJC puede contribuir a un desarrollo global genuinamente progresivo: 1. Dejar los combustibles fósiles en el suelo y, en cambio, invertir en la eficiencia energética adecuada y en la energía renovable segura, limpia y liderada por la comunidad. 2. Reducir radicalmente el consumo desperdiciado, principalmente en los países del norte, pero también por las élites del sur. 3. Enormes transferencias financieras de norte a sur, basadas en el pago de las deudas climáticas y sujetas al control democrático. Los costos de adaptación y mitigación deben pagarse redirigiendo los presupuestos militares, los impuestos innovadores y la cancelación de la deuda. 4. Conservación de recursos basada en los derechos de los pueblos indígenas sobre la propiedad y la promoción de la soberanía de las personas sobre la energía, los bosques, la tierra y el agua (Carbon Trade Watch, 2008). 
La política económica exterior se basará en el concepto de un periodo de transición del desarrollo socialista en un mar de capitalismo autocrático. Por lo tanto, trataremos de continuar el comercio para aumentar las divisas y financiar la compra de las importaciones necesarias. Como se señala en el punto anterior, los controles de capital facilitarán en la economía mundial una integración progresiva en lugar de competitiva.

Será atraído el financiamiento para el desarrollo de origen progresivo. Se buscará persuadir a los sindicatos, las municipalidades y los Estados progresistas, es decir, aquellos que son liderados y gobernados por fuerzas de izquierda para que inviertan fondos en actividades que promuevan la agenda transformadora. ${ }^{6}$

Una vez que otros Estados y regiones comienzan a emprender una transformación social progresiva, el esfuerzo debería encaminarse a promover relaciones de cooperación cercanas con ellos. Dichas relaciones serán determinadas por las necesidades y capacidades de desarrollo humano de esta colectividad internacional emergente (ALBA-TCP, 2010): 1. El comercio exterior y la inversión serán dirigidos por organismos democráticos nacionales. 2. Trato especial y diferente a las naciones con mayores necesidades de desarrollo, a quienes se les otorgará formas preferenciales de acceso a los mercados de las naciones que tienen mayores capacidades de desarrollo. 3. Cooperación y solidaridad para el desarrollo: la lucha colectiva para elevar la alfabetización de las poblaciones y la calidad de la salud. 5. El establecimiento de un fondo de emergencia social que ayude a las naciones emergentes progresistas a trascender crisis transicionales. 6. Uso de capacidades colectivas con el objeto de mejorar nuestras posiciones de negociación global en áreas

${ }^{6}$ Por ejemplo, buscaremos trabajar con movimientos como Divest London (http://divest london.org/) para reorientar las finanzas desinvertidas en actividades progresivas. 
que afectan nuestro desarrollo futuro, incluidas las normas de comercio e inversión y las normas ambientales y laborales? ${ }^{7}$

\section{Compartir y reducir el trabajo}

El capitalismo se basa en una paradoja fundamental. Los avances tecnológicos han creado una situación en la que sólo se requiere una pequeña fracción del trabajo de la mayoría de las sociedades para satisfacer sus necesidades (básicas y extendidas). Sin embargo, la propiedad privada, la acumulación competitiva de capital y la explotación de mano de obra por el capital inhabilitan este potencial. Las primeras ocho propuestas están diseñadas para transformar el control de la clase trabajadora sobre el trabajo a través de a) transferir el control sobre los medios de producción a las organizaciones de la clase trabajadora, y b) cambiar el contenido y el significado del trabajo a través de la democratización.

Los objetivos iniciales son establecer el pleno empleo para aquellos que pueden trabajar mediante la difusión y el intercambio de tareas de trabajo. Los objetivos a largo plazo son utilizar el control democrático y la dirección social de los medios de producción en aras de reducir la jornada laboral. Con la identificación de las necesidades de las comunidades individuales y de la nación en general, cada vez más será posible identificar actividades innecesarias y eliminarlas. La identificación de actividades necesarias/socialmente deseables contribuirá a la dirección de nuestra política industrial. La I+D se utilizará para establecer formas de aumentar la eficiencia y la productividad de las actividades socialmente

${ }^{7}$ Estos principios se adaptan de los estipulados por la Alianza Bolivariana para los Pueblos de Nuestra América, véase ALBA-TCP (2010). 
necesarias/deseables y reducir así el tiempo total de trabajo requerido para crearlas.

\section{Igualdad de género, nacionalismo y racismo}

Los intentos de generar desarrollo socialista fracasarán a menos que se supere la discriminación de género, étnica y racial. En los esfuerzos por trascender estas desigualdades, el movimiento de independencia kurdo ha inspirado novedosos intentos para crear un Estado autónomo en Rojava basado en la solidaridad. Los kurdos de Rojava rechazan el modelo de Estado nación que, desde su fundación, se ha sustentado en la «otredad» de minorías étnicas no nativas:

En Rojava, muchos grupos religiosos y étnicos diferentes (cristianos, yazidis, árabes, turcomanos, chechenos, armenios) conviven con la gran mayoría kurda. Al negar de manera oficial e insistente al Estado nación, y al tratar de crear estructuras administrativas que incorporen estos elementos diferentes, el modelo de Rojava otorga a las minorías un papel participativo sin precedentes en el Medio Oriente, un papel como iguales en la gestión de la polis (Aretaios, 2015).

La comunidad autónoma de Rojava ha establecido la igualdad de género como un principio organizador. Cada institución y organización tiene una cuota de 40 por ciento para la representación de mujeres, 40 por ciento para los hombres y 20 por ciento restante para cualquier sexo que reciba el mayor número de votos: «Desde la organización local más pequeña hasta el Parlamento y el gobierno, esta cuota de 40 por ciento se impone 
y, en muchos casos, existe la obligación de tener mujeres como copresidentes o vicepresidentes» (Aretaios, 2015).

\section{Cultura como desarrollo}

En el capitalismo, la producción y la participación cultural se basan en un proceso dual de degradación (de las culturas indígenas y de la clase trabajadora), y luego su reenvasado y mercantilización para la venta con fines de lucro. La cultura se establece como una esfera separada, una actividad de ocio, aislada de las actividades reproductivas sociales. Por medio de la mercantilización, la cultura se convierte en marca de distinción y diferenciación de clase (Bourdieu, 1984). Antes de esta degradación/mercantilización, representaba una forma y un foro para la participación comunitaria. El desarrollo cultural fortalecerá la propiedad social y el control de los medios de producción y la identificación democrática de las necesidades.

El desarrollo cultural se facilitará, en parte, a través de la educación avanzada para todos, basada en una pedagogía radical de los oprimidos y la concientización. Concientización es, «el proceso en el que las mujeres, no como receptoras, sino como sujetos conocedores, logran una conciencia más profunda tanto de la realidad sociocultural que da forma a sus vidas como de su capacidad para transformar esa realidad» (Freire, 1972:15).

Esta pedagogía permitirá la transformación de los objetos del desarrollo en sujetos del desarrollo. Las inversiones estatales y locales apoyarán la integración de la educación que da prioridad a la concientización acerca del funcionamiento de la planificación participativa en el nivel comunitario. Las tradiciones culturales indígenas, locales e históricas, se utilizarán para construir nuevas tradiciones educativas. Estas tradiciones contribuirán a la 
renovación cultural mediante la desalienación, la desfragmentación y la reintegración de la vida social. Se establecerán nuevos medios de televisión, radio, medios impresos y digitales para generar la difusión de la cultura de clase trabajadora y de los pueblos indígenas.

\section{Conclusión}

El capitalismo ha establecido suficiente riqueza a escala mundial para un mundo libre de pobreza, pero nunca podrá realizar ese potencial. Es un sistema de acumulación infinita de capital, de competencia, explotación, opresión y destrucción ambiental. Estas relaciones sociales destruirán el planeta, crearán nuevas formas de pobreza masiva y reproducirán megadesigualdades al mismo tiempo que propagan el sueño de bienestar para todos.

Las teorías principales del desarrollo pueden diferir en el peso que asignan a los mercados y a los Estados en el proceso de desarrollo. Sin embargo, coinciden en que la explotación laboral (y la represión) son ingredientes necesarios del desarrollo capitalista. De esa manera, se basan en una paradoja fundamental: si bien proclaman su deseo de mejorar las condiciones de los pobres del mundo, lo hacen promoviendo teorías y prácticas que legitiman y facilitan su explotación.

Los enfoques socialistas deben basarse en el reconocimiento de que la explotación laboral es un anatema para el desarrollo humano real. Desde este punto de partida, surge la pregunta de cómo se puede construir una sociedad exenta de explotación. En el presente artículo he argumentado que la construcción de una sociedad de este tipo estará cargada de tensión, incluida la muy importante dificultad de construir una nueva 
sociedad utilizando herramientas del pasado. Sin embargo, reconocer dicha tensión representa parte de la preparación mental indispensable para concebir las posibilidades del desarrollo socialista en el siglo XXI.

\section{Referencias}

Akram-Lodhi, Haroon (2015), «Accelerating towards food sovereignty», Third World Quarterly, 36(3), pp. 563-583.

Aretaios, Evangelos (15 de marzo de 2015), «The Rojava revolution», Open Democra$c y$, en https://www.opendemocracy.net/arab-awakening/evangelos-aretaios/ rojava-revolution

Bardhan, Pranab (22 de junio de 2016), «Could a basic income help poor countries?», Project Syndicate, en https://www.project-syndicate.org/commentary/ developing-country-basic-income-by-pranabbardhan-2016-06

Barker, Colin (junio de 1998), «Industrialism, capitalism, value, force and states: some theoretical remarks» (working paper), Anglo-Bulgarian Comparative History Seminar, Wolverhampton University.

Bourdieu, Pierre (1984), Distinction, Abingdon, Routledge.

Boyce, James K. y Léonce Ndikumana (28 de septiembre de 2011), «African debt: funny money and stolen lives», African Arguments, en http://africanarguments. org/2011/09/28/african-debt-funny-money-and-stolen-lives-by-james-kboyce-and-leonce-ndikumana/

Carbon Trade Watch (3 de noviembre de 2008), «Climate justice now! Statement», Carbon Trade Watch, en http://www.carbontradewatch.org/index.php?option $=$ com_content\&task=view\&id=227\&Itemid=95 
Chang, Dae-Oup (2002), «Korean labour relations in transition: authoritarian flexibility», Labour, Capital and Society, 35(1), pp, 10-40.

Clark, Brett y John Bellamy Foster (2009), «Ecological imperialism and the global metabolic rift unequal exchange and the Guano/Nitrates Trade», International Journal of Comparative Sociology, 50(3-4), pp. 311-334.

Crotty, James y Gerald Epstein (1996), «In defence of capital controls», Socialist Register, 18(32).

Edward, Peter (2006), «The ethical poverty line: a moral quantification of absolute poverty», Third World Quarterly, 27(2), pp. 377-393.

Elson, Diane (1988), «Market socialism or socialization of the market?», New Left Review, 172(3).

Freire, Paulo (1972), Pedagogy of the oppressed, New York, Penguin.

Geras, Norman (1999), «Minimum utopia: ten theses», Socialist Register, 36(36), pp. 41-52.

Gowan, Peter (1999), The global gamble: Washington's Faustian bid for world dominance, London, Verso.

Harnecker, Marta (2014), «Decentralised participatory planning based on experiences of Brazil, Venezuela and the State of Kerala, India», en http://links. org.au/node/4208

Henry, James S. (julio de 2012), «The price of offshore revisited: new estimates for «missing global private wealth, income, inequality and lost taxes», Tax Justice Network, en http://www. taxjustice.net/cms/upload/pdf/Price_of_ Offshore_Revisited_26072012.pdf

Hoy, Chris y Andy Sumner (2016), «Global poverty and inequality: is there new capacity for redistribution in developing countries?», Journal of Globalization and Development, 7(1), pp. 117-157. 
Hanlon, Joseph, Armando Barrientos y David Hulme (2012), Just give money to the poor: the development revolution from the global South, United States, Kumarian Press.

International Labour Organization (ILO) (2013), «Global wage report 2012-2013», ILO, Geneva.

Kloppenburg, Jack (2010), «Impeding dispossession, enabling repossession: biological open sourceand the recovery of seed sovereignty», Journal of Agrarian Change, 10(3), pp. 367-388.

Lebowitz, Michael A. (2015), The socialist imperative: from gotha to now, New York, NYU Press.

Mamani, Fernando H. (2010), Vivir Bien/Buen Vivir, Lima, Coordinadora Andina de Organizaciones Indígenas.

Martínez-Alier, Joan (30 de agosto de 2002), «The environmentalism of the poor: a study of ecological conflicts and valuation», working paper for the conference on The Political Economy of Sustainable Development: Environmental Conflict, Participation and Movements.

Marx, Karl (1966), The civil war in France, Peking, Foreign Languages Press, en https://www.marxists.org/archive/marx/works/1871/civil-war-france/drafts/ index

(1993), Grundrisse, London, Penguin.

McMichael, Philip D. (ed.) (1994), The global restructuring of agro-food systems, United States, Cornell University Press.

Mellor, Mary (2005), «The politics of money and credit as a route to ecological sustainability and economic democracy», Capitalism Nature Socialism, 16(2), pp. 45-60.

(2012), «Money as a public resource for development», Development, 55(1), pp. 45-53. 
Ndikumana, Léonce y James K. Boyce (2011), Africa's odious debts: how foreign loans and capital flight bled a continent, London, Zed Books.

Oxfam (2017), "An economy for the 99\%: it's time to build a human economy that benefits everyone, not just the privileged few», Oxfam, en https:// www.oxfam.org/en/research/economy-99

Rogers, Deborah y Bálint Balázs (2016), «The view from deprivation: poverty, inequality and the distribution of wealth», en Alberto D. Cimadamore, Gabriele Koehler y Thomas Pogge (eds.), Poverty and the millennium development goals, Chicago, University of Chicago Press.

South African History Online (SAHO) (2011), «The freedom charter campaign», SAHO, en http://www.sahistory.org.za/article/freedom-charter Selwyn, Benjamin (2014), The global development crisis, Cambridge, Polity. (2017), The struggle for development, Cambridge, Polity.

Shaxson, Nicholas, John Christensen y Nick Mathiason (19 de julio de 2012), «Inequality: you don't know the half of it (or why inequality is worse than we thought)», Tax Justice Network, en http://www.taxjustice.net/cms/upload/ pdf/Inequality_120722_You_dont_know_the_half_of_it.pdf

Standing, Guy (2017), Basic income: and how we can make it happen, London, Pelican.

Sumner, Andy (2016), Global poverty: deprivation, distribution and development, Oxford/New York, Oxford University Press.

Taylor, Marcus (2006), From Pinochet to the sthird way: neoliberalism and social transformation in Chile, London, Pluto.

The Bolivarian Alliance for the Peoples of our America (ALBA) (2010), «Principles of the ALBA [online]», en http://www.alba-tcp.org/en/contenido/ principles-alba 


\section{MANIFIESTO PARA EL DESARROLLO SOCIALISTA EN EL SIGLO XXI}

Trainer, Ted (1996), Towards a sustainable economy, Oxford, John Carpenter Publishing.

Tugal, Cihan (2016), The fall of the Turkish model: how the Arab uprisings brought, down islamic liberalism, London, Verso.

Weis, Tony (2010), «The accelerating biophysical contradictions of industrial capitalist agriculture», Journal of Agrarian Change, 10(3), pp. 315-341. 